\title{
Prevalência e coocorrência de Experiências Adversas na Infância: um inquérito de base escolar no município do Rio de Janeiro
}

\author{
Prevalence and co-occurrence of Adverse Childhood Experiences: \\ a school-based survey in Rio de Janeiro
}

Luciane Stochero (https://orcid.org/0000-0003-0432-2739) ${ }^{1}$

Claudia Leite Moraes (https://orcid.org/0000-0002-3223-1634) ${ }^{1}$

Emanuele Souza Marques (https://orcid.org/0000-0002-8633-7290) ${ }^{1}$

Erika Barbosa dos Santos (https://orcid.org/0000-0001-5155-7691) ${ }^{2}$

Deylaine Lourenço Pacheco (https://orcid.org/0000-0002-9287-6442) ${ }^{1}$

Michael Eduardo Reichenheim (https://orcid.org/0000-0001-7232-6745) ${ }^{1}$

Stella Regina Taquette (https://orcid.org/0000-0001-7388-3025) ${ }^{3}$

${ }^{1}$ Instituto de Medicina Social, Universidade do Estado do Rio de Janeiro. Rua São Francisco Xavier $5247^{\circ}$ andar Bloco D, Maracanã. 20550-013 Rio de Janeiro RJ Brasil. luciane.stochero@ gmail.com

${ }^{2}$ Setor de Vigilância

Epidemiológica, Secretaria Municipal de Saúde. Nova Iguaçu RJ Brasil.

${ }^{3}$ Faculdade de Ciências Médicas, Universidade do Estado do Rio de Janeiro. Rio de Janeiro RJ Brasil.

\begin{abstract}
This study aimed to estimate the prevalence of categories of adverse childhood experiences (ACE) among high school students in Rio de Janeiro, investigate the ACE co-occurrence profile, and examine the distribution of exposure to ACE according to individual, family, socioeconomic, and school characteristics. A cross-sectional study was conducted with 681 individuals selected using a complex random sampling design. Exposure to ACE categories was identified using a cross-culturally adapted version of the Childhood Trauma Questionnaire (CTQ) and direct questions. We calculated prevalence and correlation between ACE pairs and determined the co -occurrence profile of childhood adversities. The findings reveal that the most common adversities were emotional abuse and neglect and biparental family dissolution. Seventy percent of the sample reported having been exposed to at least one ACE and 9\% had been exposed to four or more. Around 20\% of respondents reported exposure to abuse and neglect and 9\% to the co-occurrence of abuse, neglect, and absence of at least one parent during childhood. The most vulnerable subgroups were girls and respondents who were born to teenage mothers, not living with both parents, studying at public schools, and from low-income families. The high prevalence and co-occurrence profile of ACE reveals the need for wide-ranging intersectoral policies designed to prevent adverse childhood experiences and provide victim support.
\end{abstract}

Key words Child abuse, Neglect, Violence, Adolescence
Resumo Este estudo tem como objetivos estimar a prevalência de Experiências Adversas na Infância (EAI), investigar o seu perfil de coocorrência e sua distribuição de acordo com características individuais, familiares, socioeconômicas e escolares de adolescentes de escolas públicas e privadas de uma Região Administrativa do Rio de Janeiro. Trata-se de um estudo seccional com 681 individuos, selecionados através de amostragem aleatória complexa. As EAI foram identificadas através do questionário QUESI e perguntas diretas. Estimou-se as prevalências e a correlação entre pares das EAI e o perfil de coocorrência das EAI. Os resultados revelaram que abuso e negligência emocionais e dissolução da família biparental foram as adversidades mais comuns. Setenta por cento da amostra sofreu pelo menos uma EAI e 9\%, quatro ou mais. Cerca de $20 \%$ vivenciou abuso e negligência e 9\% a coocorrência de abuso, negligência e ausência de pelo menos um genitor durante a infância. Os subgrupos mais vulneráveis foram: meninas, filhos de mães adolescentes, os que não moravam com ambos os pais, os de escola pública e os de baixa renda. As altas prevalências e o perfil de coocorrência das adversidades sugerem que as políticas voltadas à prevenção e ao acolhimento de vitimas sejam abrangentes e multissetoriais.

Palavras-chave Maus-tratos infantis, Negligência, Violência, Adolescência 


\section{Introdução}

As Experiências Adversas na Infância (EAI) vêm sendo definidas não só como atos de perpetração ou omissão dirigidos às crianças, mas também como um conjunto de outras condições contextuais, tais como viver em um ambiente familiar disfuncional com a presença de violência doméstica, abuso de drogas/álcool pelos pais, separação ou divórcio, morte de um ou ambos os genitores, dentre outros ${ }^{1,2}$. Estas experiências adversas comumente coocorrem e expõe a criança a várias adversidades consecutivas ou mesmo simultâneas. Estudos indicam que quanto maior o número de experiências adversas vivenciadas, maior o efeito negativo no desenvolvimento do indivíduo ${ }^{1-4}$.

As prevalências de EAI em países de alta, média e baixa renda são elevadas em todos os continentes ${ }^{2,5-8}$. Porém, podem variar de acordo com a definição conceitual adotada, a estratégia utilizada para a sua detecção e as características da amostra estudada ${ }^{9,10}$. Fatores socioeconômicos e culturais, incluindo grau de desigualdade, de desemprego e estereótipos de gênero, também desempenham um importante papel na distribuição das prevalências ${ }^{11,12}$. Estudo realizado em 21 países de diferentes níveis de renda em 2010, por exemplo, indicou que $66,2 \%$ dos indivíduos de países de baixa renda haviam reportado pelo menos uma EAI, enquanto 59,3\% de países de alta renda haviam indicado a situação. Já as prevalências de coocorrência de quatro EAI foi de 3,1\% nos países de baixa renda e 5,0\% nos de alta renda ${ }^{2}$. No Brasil, a literatura sobre o tema ainda é escassa. Destaca-se o estudo realizado com adultos residentes em São Paulo e 38 cidades próximas em 2016 indicando que 53,6\% dos entrevistados haviam sofrido pelo menos uma EAI durante a infância e adolescência ${ }^{13}$. No mesmo ano, estudo desenvolvido em Pelotas, no Rio Grande do Sul, identificou que 85\% dos indivíduos de até 18 anos de idade haviam sofrido pelo menos uma EAI, e que 7,1\% das meninas e 3,2\% dos meninos haviam relatado quatro ou mais adversidades ${ }^{6}$.

A relevância do tema também se deve às diversas consequências negativas à saúde gerada pela exposição contínua e cumulativa às EAI. Estas podem se dar ainda na infância através de distúrbios do sono ${ }^{14}$, atrasos no desenvolvimento cognitivo $^{15}$ e demais problemas de saúde física ${ }^{16}$. $\mathrm{Na}$ adolescência, levando ao consumo de álcool e drogas ${ }^{17}$, ao envolvimento em situações de violência ${ }^{18}$, à iniciação sexual precoce e insegura ${ }^{5,19}$;
$\mathrm{Na}$ idade adulta, promovendo o consumo abusivo de álcool e drogas ${ }^{20}$, comportamento sexual inseguro ${ }^{5}$, problemas de saúde mental ${ }^{21}$ como a depressão ${ }^{22,23}$, e de ordem física ${ }^{24}$, como problemas cardiovasculares, entre outros ${ }^{25}$.

$\mathrm{Na}$ adolescência, as consequências prejudiciais também vão além das repercussões na saúde. No contexto escolar, por exemplo, ressalta-se que os expostos a traumas familiares têm maior dificuldade de adaptação, demonstram menor interesse durante as aulas e maiores taxas de suspensão e expulsão em razão de condutas agressivas, o que pode levar ao abandono escolar ${ }^{26,27}$. A escola também pode servir de palco para a ocorrência de algumas EAI, tais como o bullying e o isolamento/rejeição entre pares ${ }^{28,29}$. Por outro lado, o ambiente escolar é um local privilegiado para a prevenção, detecção, intervenção e apoio aos que vivenciaram tais situações. Neste sentido, a escola pode criar estratégias de enfrentamento e desenvolvimento de habilidades de resiliência, na qual as crianças e os adolescentes se sintam protegidos, incentivados e engajados no processo de transformaçãa ${ }^{26,27}$.

Em virtude das altas prevalências de EAI no mundo, de suas graves consequências à saúde e em outras esferas da vida durante a adolescência e da escassez de estudos sobre o tema no Brasil, parece oportuno o desenvolvimento de pesquisas que visem conhecer melhor o problema em nosso contexto. Este estudo tem esta intenção. Seus objetivos são: estimar a prevalência de abuso emocional, físico e sexual, negligência emocional e física, morte ou perda de contato com os genitores e dissolução da família biparental na infância; investigar o perfil de coocorrência destas EAI; e analisar a distribuição dos eventos segundo características individuais, da estrutura familiar, do contexto socioeconômico e relativas à escola de adolescentes escolares da IX Região Administrativa (RA) do município do Rio de Janeiro.

\section{Métodos}

\section{Desenho e cenário de estudo}

Trata-se de um estudo seccional de base escolar com alunos do $2^{\circ}$ ano do Ensino Médio de escolas públicas e privadas pertencentes a IX RA do município do Rio de Janeiro, que envolve os bairros do Maracanã, Vila Isabel, Andaraí e Grajaú. De acordo com o censo de 2010, esta região apresentava cerca de 190 mil habitantes, sendo aproximadamente 17 mil crianças e 22 mil ado- 
lescentes, pertencentes a famílias com diferentes níveis de renda, com renda média per capita de 3,6 salários mínimo ${ }^{30}$.

\section{População fonte, tamanho amostral e estratégia de seleção de participantes}

A população fonte concerne 1.470 estudantes regularmente matriculados no $2^{\circ}$ ano do Ensino Médio em 2016, em cinco escolas públicas e sete privadas, distribuídos em 52 turmas. Os participantes foram selecionados através de uma amostragem aleatória complexa, estratificada por tipo de gestão (pública ou privada) e o turno (diurno ou noturno) da escola. As turmas que participariam da pesquisa foram sorteadas com probabilidade de seleção proporcional ao tamanho das escolas. Todos os alunos das turmas sorteadas foram convidados a participar da pesquisa. Para o cálculo do tamanho amostral, usou-se a equação: $\mathrm{n}-Z^{2}{ }_{1}{ }_{\alpha / 2} P(1-P) / d^{2}$.

Onde $\alpha$ é o nível de significância, $Z$ é o quantil da distribuição normal, $\mathrm{P}$ é a prevalência esperada do principal desfecho de interesse na pesquisa de fundo (violência nos relacionamentos amorosos) e d a margem de erro, considerando os seguintes valores: $\alpha=0,05, \mathrm{P}=0,25 \mathrm{e} \mathrm{d}=0,05$. $\mathrm{O}$ tamanho amostral estimado inicialmente foi multiplicado por dois para permitir que os resultados fossem estratificados por sexo (31). O estudo de fundo incluiu 721 indivíduos. Para o presente estudo, excluiu-se 21 indivíduos com 20 anos ou mais, 14 que se autorreferiram indígenas e 7 asiáticos, em função do número reduzido de participantes com estas características, resultando em uma amostra de 681 alunos.

\section{Coleta de dados e instrumentos de aferição das variáveis de interesse}

A coleta de dados ocorreu no período de setembro de 2016 a fevereiro de 2017 e foi coordenada por equipe previamente treinada. As informações foram coletadas através de questionário multitemático autoaplicado em salas de aula.

\section{Experiências Adversas na Infância}

O estudo avaliou a ocorrência de abuso emocional, físico e sexual; negligência emocional e física; morte ou perda de contato com os genitores e dissolução da família biparental, eventos escolhidos dentre as EAI indicadas por Felitti et al. ${ }^{1} \mathrm{e}$ Kesller et al. ${ }^{2}$. Os conceitos de abuso emocional, físico e sexual se baseiam nos propostos pela Organização Mundial da Saúde (OMS) em seu re- latório sobre violência e saúde ${ }^{32}$ enquanto que os de negligência emocional e física se baseiam nos propostos por Bernstein e Fink ${ }^{33}$. As experiências adversas de abusos e negligência foram aferidas utilizando-se o Questionário Sobre Traumas na Infância $(\mathrm{QUESI})^{34}$, versão brasileira e adaptada transculturalmente do instrumento Childhood Trauma Questionnaire (CTQ) ${ }^{33}$.O QUESI compreende cinco dimensões, com cinco itens cada, que cobrem os tipos de violência citadas acima, além de três itens extras que visam a facilitação da entrevista. As opções de respostas variam em uma escala de um a cinco pontos (nunca; poucas vezes; às vezes; muitas vezes; sempre). $\mathrm{O}$ indivíduo foi considerado um caso positivo para as estimativas de prevalência de cada uma das violências na infância quando respondeu "poucas vezes", "às vezes", "muitas vezes" ou "sempre" a pelo menos um dos itens da referida subescala.

A ocorrência de morte ou perda de contato com os genitores durante a infância foi abordada através de perguntas diretas: "Seu pai está vivo?" e "Sua mãe está viva?". Os indivíduos que responderam negativamente às questões ou que relataram perda de contato com os genitores antes dos 10 anos de idade, foram considerados positivos para estas adversidades. A adversidade dissolução da família biparental foi caracterizada quando o adolescente referiu não ter morado com a mãe e o pai também no mesmo período.

\section{Variáveis demográficas e socioeconômicas}

A variável raça/cor utilizou as categorias do Instituto Brasileiro de Geografia e Estatística (IBGE). A dimensão estrutura familiar contemplou informações sobre a idade da mãe ao nascimento do adolescente e com quem mora o adolescente atualmente. Para caracterizar o status socioeconômico da família, foram utilizadas: escolaridade da mãe e o poder de compra da família, mensurado através do Critério de Classificação Econômica Brasil (CCEB), versão 2015 ${ }^{35}$. As demais variáveis são autoexplicativas e se encontram na primeira tabela da seção de resultados.

\section{Análise de dados}

A análise de dados considerou a estrutura de amostragem complexa. Foram estimadas as prevalências de EAI na amostra como um todo e em subgrupos de acordo com as características individuais, familiares, socioeconômicas e escolares da população de estudo. O perfil de coocorrência das EAI foi investigado com três estratégias. A primeira contemplou a contagem do número de adversidades na infância. Na segunda aborda- 
gem, avaliou-se o grau de correlação entre pares de adversidades. Como as variáveis são dicótomas (sim/não), foi utilizado o estimador Tau de Kendall, indicado para variáveis ordinais e adequado para amostras com tamanho reduzido ${ }^{36}$. O perfil de coocorrências também foi representado graficamente utilizando o Diagrama de Venn, agrupando-se as experiências em três conjuntos: abusos (emocional e/ou físico e/ou sexual), negligências (emocional e/ou física) e ausência de pelo menos um genitor (morte ou perda de contato com os genitores e/ou dissolução da família biparental). Utilizou-se o teste Qui-Quadrado $\left(\chi^{2}\right)$ para avaliar a homogeneidade das prevalências nos subgrupos populacionais, considerando o p-valor menor que 0,05 para a identificação das diferenças estatisticamente significativas. As análises foram realizadas no software Stata $15^{37}$.

\section{Questões éticas}

O estudo de fundo foi aprovado pelo Comitê de Ética em Pesquisa da Universidade do Estado do Rio de Janeiro, tendo o Certificado de Apresentação para Apreciação Ética e pela Secretaria de Estado de Educação. O Termo de Consentimento Livre e Esclarecido foi assinado pelos pais ou responsáveis e pelos alunos.

\section{Resultados}

Como visto na Tabela 1, a amostra contemplou proporções semelhantes de meninos e meninas. A maioria declarou ser da raça/cor branca. Mais de $70 \%$ das mães tinham entre 20 e 35 anos de idade no momento do nascimento do(a) filho(a). Mais da metade da amostra não residia com a família biparental à época da entrevista. A maioria das mães tinha baixa escolaridade. As famílias pertencentes às classes econômicas alta e média (Classes A e B) representam cerca de $70 \%$ da amostra e apenas cerca $1 \%$ faz parte da classe mais baixa (E). A maior parte dos entrevistados estudava na rede particular de ensino em turno diurno.

Como pode ser visto na Tabela 2, as EAI mais reportadas foram o abuso emocional, negligência emocional e a dissolução da família biparental. As meninas e os filhos/as de mães com nível médio foram as vítimas mais comuns de abuso emocional. A prevalência de abuso físico foi maior entre os que não moram com ambos os pais, bem como entre aqueles que a mãe também tem um nível médio de escolaridade. Enquanto o abuso sexual foi mais frequente entre as meninas. A negligência emocional foi mais frequente entre
Tabela 1. Perfil da amostra de estudo. Inquérito de base escolar IX RA do município do Rio de Janeiro, RJ.

\begin{tabular}{lllll}
\hline $\begin{array}{c}\text { Características da } \\
\text { amostra }\end{array}$ & $\mathbf{n}^{*}$ & $\mathbf{n}^{\text {exp**}}$ & $\%$ & $($ IC 95\%) \\
\hline $\begin{array}{l}\text { Características do } \\
\text { indivíduo }\end{array}$ & & & & \\
Sexo & & & & \\
$\quad$ Feminino & 367 & 676 & 53,2 & $(46,8-59,5)$ \\
$\quad$ Masculino & 314 & 594 & 46,8 & $(40,4-53,2)$ \\
Raça/cor & & & & \\
$\quad$ Branca & 325 & 679 & 53,7 & $(46,1-61,2)$ \\
$\quad$ Preta & 116 & 189 & 14,9 & $(12,1-18,3)$ \\
$\quad$ Parda & 236 & 395 & 31,3 & $(25,7-37,5)$ \\
\end{tabular}

\section{Estrutura familiar}

Idade da mãe no

nascimento

$\begin{array}{lrrrr}\text { Até } 19 \text { anos } & 96 & 158 & 13,4 & (10,5-16,9) \\ 20-35 \text { anos } & 473 & 866 & 73,3 & (66,3-79,3) \\ \text { Maior que 35 } & 64 & 157 & 13,3 & (7,90-21,5)\end{array}$

anos

Com quem mora o

adolescente

Com pai e mãe $\quad 297 \quad 563 \quad 44,6 \quad(41,0-48,2)$

Apenas com mãe/ $222 \quad 436 \quad 34,5 \quad(31,5-37,8)$

apenas com pai

$\begin{array}{lllll}\text { Com mãe e } & 112 \quad 192 & 15,2 & (12,9-17,8)\end{array}$

padrasto/ com pai

e madrasta

Outros

$46 \quad 71,4 \quad 5,65 \quad(4,09-7,70)$

\section{Características}

socioeconômicas

Escolaridade

materna

$\begin{array}{lrrrr}\text { Baixa (0-8 anos) } & 407 & 661 & 54,4 & (46,2-62,2) \\ \text { Média (9-12 anos) } & 77 & 167 & 13,7 & (11,4-16,3) \\ \text { Alta (>12 anos) } & 162 & 389 & 32,0 & (25,1-39,9) \\ \text { CCEB } & & & & \\ \text { A } & 91 & 208 & 17,5 & (13,6-22,2) \\ \text { B } & 321 & 655 & 55,1 & (51,4-58,7) \\ \text { C } & 204 & 313 & 26,3 & (21,7-22,1) \\ \text { D-E } & 11 & 14 & 1,2 & (0,60-2,30)\end{array}$

\section{Características da}

Escola

Gestão da escola

$\begin{array}{lllll}\text { Pública } & 373 & 458 & 36,0 & (31,3-41,2) \\ \text { Privada } & 308 & 653 & 64,0 & (58,8-68,8)\end{array}$

Turno de aula

\begin{tabular}{lrrrr} 
Diurno & 583 & 1159 & 91,3 & $(89,1-93,1)$ \\
Noturno & 98 & 110 & 8,70 & $(6,94-10,9)$ \\
\hline
\end{tabular}

* População de estudo (sem considerar os pesos amostrais). ** Amostra expandida a partir dos pesos amostrais. IC95\%: Intervalo de Confiança a 95\%. CCEB = Critério de Classificação Econômica Brasil.

Fonte: Pesquisa Estupro de vulnerável e outras violências contra adolescentes e jovens do sexo feminino, 2015. 
Tabela 2. Prevalências de Experiências Adversas na Infância por categoria de acordo com o perfil sócio demográfico da amostra. Inquérito de base escolar IX RA do município do Rio de Janeiro, RJ.

\begin{tabular}{|c|c|c|c|c|c|c|c|}
\hline \multirow{2}{*}{ Características da amostra } & $\mathrm{AE}$ & AF & AS & $\mathrm{NE}$ & NF & MPCG & DFB \\
\hline & $\%^{*}$ & $\%^{*}$ & $\%^{*}$ & $\%^{*}$ & $\%^{*}$ & $\%^{*}$ & $\%^{*}$ \\
\hline Amostra total & 41,7 & 16,8 & 10,5 & 35,2 & 16,7 & 5,10 & 24,3 \\
\hline \multicolumn{8}{|l|}{ Sexo } \\
\hline Feminino & $46,8^{\star *}$ & 17,7 & $14,3^{* *}$ & 36,0 & 15,1 & 5,00 & 25,2 \\
\hline Masculino & 35,8 & 15,8 & 6,16 & 34,2 & 18,5 & 5,18 & 23,3 \\
\hline \multicolumn{8}{|l|}{ Raça/cor } \\
\hline Branca & 45,9 & 15,3 & 12,5 & 35,9 & 16,0 & 4,62 & 25,2 \\
\hline Preta & 40,0 & 17,0 & 8,94 & 31,1 & 18,5 & 7,59 & 21,2 \\
\hline Parda & 36,2 & 19,6 & 7,89 & 35,2 & 16,9 & 4,70 & 24,6 \\
\hline \multicolumn{8}{|l|}{ Idade da mãe ao nascimento } \\
\hline Até 19 anos & 47,4 & 22,7 & 8,74 & $43,9^{* *}$ & 19,8 & 7,89 & 30,9 \\
\hline De 20 a 35 anos & 39,2 & 16,3 & 10,2 & 32,4 & 17,0 & 3,60 & 21,2 \\
\hline Maior que 35 anos & 56,9 & 14,3 & 18,1 & 47,0 & 12,3 & 1,84 & 26,1 \\
\hline \multicolumn{8}{|l|}{ Com quem mora o adolescente } \\
\hline Com pai e mãe & 36,5 & $12,4^{\star *}$ & 9,37 & $26,6^{\star *}$ & 15,4 & - & - \\
\hline $\begin{array}{l}\text { Apenas com mãe/apenas com } \\
\text { pai }\end{array}$ & 47,1 & 19,6 & 9,82 & 41,0 & 15,6 & $7,55^{\star *}$ & $40,8^{\star *}$ \\
\hline Mãe e padrasto/ pai e madrasta & 46,6 & 23,1 & 14,8 & 41,9 & 20,4 & 7,34 & 52,9 \\
\hline Outros & 40,2 & 18,6 & 12,3 & 51,7 & 22,7 & 24,0 & 38,1 \\
\hline \multicolumn{8}{|l|}{ Escolaridade materna } \\
\hline Baixa (0-8 anos) & $36,7^{\star \star}$ & $13,9^{* *}$ & 10,0 & 36,0 & 17,0 & 4,30 & 19,5 \\
\hline Média (9-12 anos) & 51,7 & 31,0 & 12,3 & 45,2 & 10,5 & 1,40 & 30,2 \\
\hline Alta (>12 anos) & 47,1 & 15,7 & 11,0 & 29,7 & 18,0 & 4,10 & 26,5 \\
\hline \multicolumn{8}{|l|}{ CCEB } \\
\hline A & 51,5 & 16,8 & 10,8 & 31,2 & 19,3 & 4,02 & 25,7 \\
\hline B & 42,0 & 15,9 & 12,0 & 36,2 & 12,6 & 4,65 & 23,4 \\
\hline $\mathrm{C}$ & 38,5 & 19,4 & 8,89 & 36,0 & 17,6 & 4,81 & 24,0 \\
\hline D-E & 23,6 & 26,6 & 0,00 & 51,2 & 54,1 & 11,9 & 20,3 \\
\hline \multicolumn{8}{|l|}{ Gestão da escola } \\
\hline Pública & 38,0 & 18,5 & 8,01 & 35,1 & $22,2^{\star \star}$ & 6,63 & 20,9 \\
\hline Privada & 43,9 & 15,9 & 11,8 & 35,3 & 13,6 & 4,18 & 26,2 \\
\hline \multicolumn{8}{|l|}{ Turno de aula } \\
\hline Diurno & 42,7 & 17,0 & 10,7 & 34,1 & $15,2^{\star \star}$ & 4,83 & 24,6 \\
\hline Noturno & 32,1 & 15,0 & 7,73 & 46,2 & 32,2 & 7,39 & 21,2 \\
\hline
\end{tabular}

$\mathrm{AE}=$ Abuso Emocional; $\mathrm{AF}=$ Abuso Físico; $\mathrm{AS}=\mathrm{Abuso}$ Sexual; $\mathrm{NE}=$ Negligência Emocional; $\mathrm{NF}=$ Negligência Física; $\mathrm{MPCG}=\mathrm{Morte}$ ou perda de contato com os genitores; $\mathrm{DFB}=$ Dissolução da família biparental. ${ }^{\star}$ Prevalência estimada considerando os pesos amostrais. ${ }^{*}$ P-valor $<0,05$. IC95\% $=$ Intervalo do confiança a 95\%. CCEB = Critério de Classificação Econômica Brasil.

Fonte: Pesquisa Estupro de vulnerável e outras violências contra adolescentes e jovens do sexo feminino, 2015.

aqueles cujas mães haviam dado à luz na adolescência ou que haviam tido o filho depois dos 35 anos, entre os adolescentes que não moram com os pais e entre aqueles que estudam no turno noturno. A negligência física foi mais prevalente em quem estuda em escola pública, no turno noturno e entre aqueles das classes econômicas D ou E.

Cerca de $70 \%$ da amostra refere ter sofrido pelo menos uma EAI (Tabela 3), a proporção diminui conforme aumenta o número de EAI consideradas. Chama a atenção o padrão cumulativo das situações de vulnerabilidade, já que grande parte dos entrevistados refere a vivência de duas ou mais adversidades, além de quase $10 \%$ ter referido quatro ou mais EAI. Os adolescentes cujas mães tiveram seus filhos nas faixas etárias extremas ( $<20$ anos e $>35$ anos) tenderam a um maior número de EAI. Por outro lado, há uma menor prevalência de experiências adversas entre os que moram com ambos os pais. 
Tabela 3. Número de Experiências Adversas na Infância de acordo com o perfil da amostra de estudo. Inquérito de base escolar IX RA do município do Rio de Janeiro- RJ

\begin{tabular}{|c|c|c|c|c|c|c|}
\hline \multirow{2}{*}{ Características da amostra } & 0 EAI & 1 EAI & 2 EAI & 3 EAI & 4+ EAI & \multirow{2}{*}{ P-valor } \\
\hline & $\%^{*}$ & $\%^{*}$ & $\%^{*}$ & $\%^{*}$ & $\%^{*}$ & \\
\hline Amostra total & 29,2 & 28,0 & 17,7 & 16,3 & 8,9 & \\
\hline \multicolumn{7}{|l|}{ Sexo } \\
\hline Feminino & 28,6 & 26,0 & 18,6 & 16,4 & 10,3 & \multirow[t]{2}{*}{0,599} \\
\hline Masculino & 29,9 & 30,5 & 16,6 & 16,2 & 7,14 & \\
\hline \multicolumn{7}{|l|}{ Raça/cor } \\
\hline Branca & 29,2 & 24,9 & 18,1 & 18,6 & 9,22 & \multirow[t]{3}{*}{0,624} \\
\hline Preta & 26,4 & 34,1 & 19,9 & 12,7 & 6,84 & \\
\hline Parda & 30,7 & 29,6 & 15,8 & 14,5 & 9,41 & \\
\hline \multicolumn{7}{|l|}{ Idade da mãe no nascimento } \\
\hline Até 19 anos & 24,5 & 29,8 & 16,1 & 10,5 & 19,1 & \multirow[t]{3}{*}{0,007} \\
\hline De 20 a 35 anos & 29,9 & 31,0 & 17,9 & 14,1 & 6,99 & \\
\hline Maior que 35 anos & 28,0 & 11,7 & 17,9 & 35,2 & 7,07 & \\
\hline \multicolumn{7}{|l|}{ Com quem mora o adolescente } \\
\hline Com pai e mãe & 44,2 & 28,0 & 14,7 & 9,15 & 3,93 & \multirow[t]{3}{*}{0,000} \\
\hline Apenas com mãe/ apenas com pai & 18,0 & 28,2 & 20,4 & 24,1 & 9,37 & \\
\hline Mãe e padrasto/pai e madrasta & 17,0 & 25,7 & 22,3 & 16,0 & 18,9 & \\
\hline \multicolumn{7}{|l|}{ Escolaridade materna } \\
\hline Baixa (0-8 anos) & 33,3 & 28,7 & 16,3 & 13,6 & 8,10 & \multirow[t]{3}{*}{0,217} \\
\hline Média (9-12 anos) & 15,4 & 27,0 & 27,9 & 20,8 & 8,89 & \\
\hline $\operatorname{Alta}(>12$ anos $)$ & 28,3 & 28,7 & 15,0 & 19,9 & 8,12 & \\
\hline \multicolumn{7}{|l|}{ CCEB } \\
\hline A & 26,3 & 24,9 & 18,1 & 21,6 & 9,13 & \multirow[t]{4}{*}{0,845} \\
\hline B & 31,0 & 27,1 & 18,0 & 15,5 & 8,36 & \\
\hline $\mathrm{C}$ & 29,8 & 29,9 & 16,0 & 14,2 & 10,0 & \\
\hline $\mathrm{D}-\mathrm{E}$ & 19,6 & 30,2 & 7,83 & 34,0 & 8,31 & \\
\hline \multicolumn{7}{|l|}{ Gestão da escola } \\
\hline Pública & 30,0 & 28,9 & 17,3 & 14,0 & 9,70 & \multirow[t]{2}{*}{0,800} \\
\hline Privada & 28,8 & 27,3 & 17,9 & 17,6 & 8,40 & \\
\hline \multicolumn{7}{|l|}{ Turno de aula } \\
\hline Diurno & 29,5 & 27,6 & 18,1 & 16,0 & 8,73 & \multirow[t]{2}{*}{0,696} \\
\hline Noturno & 26,5 & 30,6 & 12,9 & 19,8 & 10,3 & \\
\hline
\end{tabular}

* Prevalência estimada com pesos amostrais. IC95\% = intervalo do confiança a 95\%. CCEB = Critério de Classificação Econômica Brasil. $0 \mathrm{EAI}$ = indivíduos que não vivenciaram nenhuma experiência adversa; $1 \mathrm{EAI}=$ indivíduos que vivenciaram apenas uma experiência adversa; $2 \mathrm{EAI}=$ indivíduos que vivenciaram duas experiências adversas; $3 \mathrm{EAI}=$ indivíduos que vivenciaram três experiências adversas; 4+ EAI: indivíduos que vivenciaram quatro ou mais experiências adversas.

Fonte: Pesquisa Estupro de vulnerável e outras violências contra adolescentes e jovens do sexo feminino, 2015.

Como pode ser visto na Tabela 4, a maior parte das correlações entre pares de EAI se mostrou positiva e estatisticamente significativa, indicando, mais uma vez, a tendência a um acúmulo de EAI. Nota-se que as três formas de abuso se correlacionam positivamente entre si e com as duas formas de negligência, apesar de não apresentarem correlações com a perda dos pais ou dissolu- ção da família biparental. Ademais, a negligência emocional se correlaciona positivamente com a dissolução da família biparental, enquanto esta última se correlaciona positivamente com a morte ou perda de contato com os genitores.

O perfil de coocorrência das EAI é apresentado na Figura 1. Observa-se que apenas 30\% da amostra relata não ter vivenciado nenhuma das 
Tabela 4. Correlação de Kendall entre pares de Experiências Adversas na Infância por categorias na amostra. Inquérito de base escolar IX RA do município do Rio de Janeiro, RJ.

\begin{tabular}{lrrrrrrr}
\hline & \multicolumn{1}{c}{ AE } & \multicolumn{1}{c}{ AF } & \multicolumn{1}{c}{ AS } & NE & NF & MPCG & DFB \\
\hline AE & 1,0000 & & & & & & \\
AF & $0,2192^{* *}$ & 1,0000 & & & & & \\
AS & $0,2193^{* *}$ & $0,1378^{* *}$ & 1,0000 & & & & \\
NE & $0,3874^{* *}$ & $0,1475^{* *}$ & $0,2079^{* *}$ & 1,0000 & & & \\
NF & $0,0944^{*}$ & $0,1003^{*}$ & $0,1288^{* *}$ & $0,2029^{* *}$ & 1,0000 & & \\
MPCG & $-0,0210$ & $-0,0549$ & 0,0604 & 0,0066 & 0,0729 & 1,0000 & \\
DFB & 0,0586 & 0,0684 & 0,07453 & $0,0932^{*}$ & $-0,0001$ & $0,3159^{* *}$ & 1,0000 \\
\hline
\end{tabular}

$\mathrm{AE}=$ Abuso Emocional; $\mathrm{AF}=\mathrm{Abuso}$ Físico; $\mathrm{AS}=\mathrm{Abuso}$ Sexual; $\mathrm{NE}=$ Negligência Emocional; $\mathrm{NF}=\mathrm{Negligên}$ cia Física; $\mathrm{MPCG}=\mathrm{Morte}$ ou perda de contato com os genitores; $\mathrm{DFB}=$ Dissolução da família biparental. ${ }^{\star} \mathrm{P}$-valor $<0.05$. ${ }^{\star \star} \mathrm{P}$-valor $<0.01$. Estimador: Tau-b de Kendall.

Fonte: Pesquisa Estupro de vulnerável e outras violências contra adolescentes e jovens do sexo feminino, 2015.

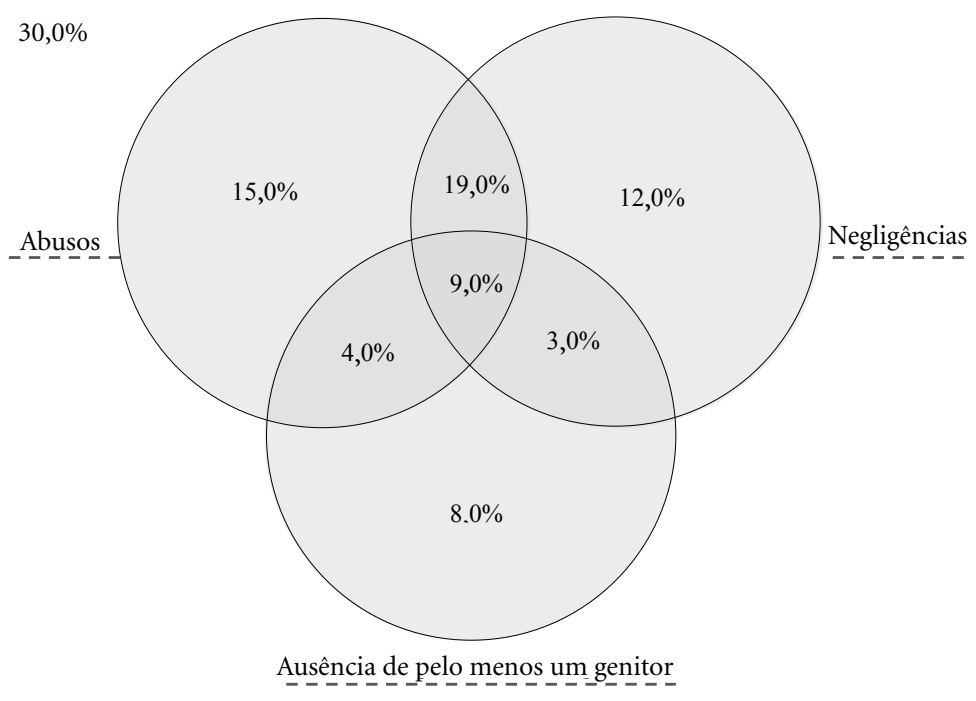

Figura 1. Coocorrência de Experiências Adversas na Infância. Inquérito de base escolar IX RA do Município do Rio de Janeiro, RJ.

Nota: Abusos (emocional e/ou físico e/ou sexual), negligências (emocional e/ou física) e ausência de pelo menos um genitor (morte ou perda de contato com os genitores e/ou dissolução da família biparental).

Fonte: Pesquisa Estupro de vulnerável e outras violências contra adolescentes e jovens do sexo feminino, 2015.

EAI investigadas, enquanto 35\% da mesma refere ter vivenciado situações pertencentes a dois ou três dos grupos de adversidades.

\section{Discussão}

A proteção e garantia dos direitos fundamentais na infância e adolescência integram os princí- pios fundamentais legitimados pela Declaração Universal dos Direitos da Criança (1959) e pela Convenção Internacional de Direitos da Criança e Adolescente ${ }^{38}$. No Brasil, o Estatuto da Criança e do Adolescente (ECA) indica no artigo quinto que nenhuma criança ou adolescente será objeto de qualquer forma de negligência, discriminação, exploração, violência, crueldade e opressã $0^{39}$. Porém, os resultados do estudo indicam que não estamos 
conseguindo garantir estes direitos para muitas das nossas crianças e adolescentes da cidade do Rio de Janeiro, na medida em que grande parte dos entrevistados referiu ter sido vítima de violências de diferentes naturezas. Também chama a atenção a grande parcela vivenciou situações de perda de contato com um dos genitores, seja por morte ou separação dos pais; e um padrão cumulativo de adversidades na infância, tornando esta população ainda mais vulnerável às suas consequências de curto, médio e longo prazo.

Sejam isoladas ou ocorrendo em conjunto, as altas prevalências de EAI chamam ainda mais a atenção ao se considerar que foram focalizadas apenas as adversidades ocorridas até os dez anos de idade, por ser este o período mais sensível às mesmas $^{40,41}$, o que difere da maioria dos estudos que estimam a prevalência dos eventos na faixa etária de 0 a 19 anos. Ao incluir também a adolescência no período de recordatório, tais pesquisas não só ampliam a probabilidade de ocorrência dos eventos aqui analisados, mas também alargam o leque de experiências investigadas, incluindo aquelas que ocorrem majoritariamente na adolescência, tais como o bullying, a rejeição $\mathrm{e}$ as agressões físicas por pares, a violência no namoro, dentre outras ${ }^{42}$. Desta forma, supõe-se que se ampliássemos o período de recordatório para incluir a adolescência, nossas estimativas seriam ainda mais elevadas.

Como apontado anteriormente, dentre as sete EAI investigadas chamam a atenção as altas prevalências do abuso e negligência emocionais e da dissolução da família biparental. Dentre estes, o abuso emocional se destaca por ser a adversidade mais frequente, especialmente entre meninas e entre aqueles cujas mães eram adolescentes no momento de seu nascimento. Estudos anteriores também sugerem que este tipo de abuso seja preferencialmente perpetrado contra meninas, podendo este comportamento estar associado a ambientes familiares rígidos, controladores, superprotetores ou caóticos ${ }^{32,43-45}$. A maior frequência entre mães jovens também é ressaltada por outros autores que relacionam a prática ao baixo apoio psicossocial disponível para as mães adolescentes $^{43}$.

Os resultados também confirmam outros achados ao identificar que aqueles que não moram com ambos os pais reportam maior frequência de abuso físico. Neste contexto, ressalta-se que o padrasto, madrasta ou familiares como tios, avós ou primos que coabitam com a criança na ausência de um dos genitores costumam ser os principais autores da violência ${ }^{32,46}$. A maior prevalência deste tipo de abuso em famílias composta por mães com menor escolaridade também é indicada em outros estudos temáticos. $\mathrm{O}$ fato habitualmente associado a menor capacidade argumentativa materna na resolução de conflitos e à falta de conhecimento de outras práticas disciplinares; A crença de que as práticas educativas parentais devem incluir atos de violência física quando necessários, bem como situações de hostilidade intensa também contribuem para as altas prevalências do problema ${ }^{32,47,48}$.

Também chama-nos atenção a alta prevalência de abuso sexual, que apesar de ser a forma de violência menos frequente entre os entrevistados, tem severas consequências na saúde física e mental dos indivíduos. Ademais, suas consequências negativas também se manifestam em outras esferas da vida podendo levara problemas no desempenho escolar, gravidez indesejada, redução da produtividade no trabalho, dentre outros ${ }^{32,49,50}$. Tal adversidade também foi mais frequente entre meninas, principais vítimas da cultura machista, patriarcal e adultocêntrica de nossa sociedade, também expressa na organização e estrutura de poder familiar. Tais determinantes sociais não só impõem que meninas sejam as vítimas preferenciais, mas que estas permaneçam em silêncio e não peçam ajuda, perpetuando sua vitimização $0^{6,32,51,52}$.

Os casos de negligência emocional ocorreram em boa parte entre aqueles que não moravam com ambos os pais, corroborando outros achados da literatura ${ }^{32,53}$. Já a negligência física esteve mais presente entre adolescentes das classes econômicas mais baixas, estudantes de escola pública e que frequentam o turno noturno, $o$ que confirma estudos anteriores com relação a maior frequência do problema entre famílias de camadas sociais e econômicas menos favorecidas. Nestas famílias, a dificuldade em prover as necessidades físicas da criança pode ser consequência de dificuldades financeiras, longas jornadas de trabalho, maior número de filhos e mesmo problemas de saúde mental, mais frequentes neste subgrupo $^{32,53,54}$. Se por um lado há que se considerar que, muitas vezes, a negligência física decorre de situações de pobreza e vulnerabilidade social, por outro, ela também pode ser resultado de déficits de habilidades parentais em prover o que a criança necessita (saúde, educação, afeto, nutrição, abrigo e segurança), mesmo quando os pais têm condições financeiras de fazê-lo ${ }^{32}$.

A morte ou perda de contato com os pais foi menos frequente em nosso estudo do que em estudos anteriores realizados no Brasil, provavel- 
mente por estes últimos utilizarem um período mais longo de recordatório, englobando também a adolescência ${ }^{2,6,7,13}$. Entretanto, a questão merece destaque por se configurar como um dos cenários de perda mais duros na infância, podendo trazer importantes consequências negativas na vida emocional e afetiva ${ }^{55,56}$. Apesar da dissolução da família biparental não ter, necessariamente, um impacto negativo na vida da criança, famílias monoparentais costumam ter menor renda per capita e menor grau de supervisão parental, tornando as crianças mais vulneráveis a riscos psicossociais e econômicos ${ }^{57,58}$. Quando conflituosa, a separação pode ser uma fonte de sentimentos de abandono e rejeição para os filhos, sendo que alguns casos evoluem para a alienação parenteral, cenário de muita dor para a criança, pois esta perde o contato com um dos genitores ${ }^{59,60}$. A recomposição das famílias com novos parceiros pode reduzir estas consequências, ainda que também possa gerar novos desafios para a criança. A sensação de não pertencimento à nova família, por se sentir traindo um dos pais biológicos ao criar laços com o novo integrante, é apenas um exemplo destas situações ${ }^{61}$.

Não obstante às altas prevalências das diferentes EAI, muitas vezes, tais adversidades coocorrem, intensificando ainda mais suas consequências desfavoráveis para o crescimento e desenvolvimento infantil. $\mathrm{O}$ acúmulo de adversidades em filhos(as) de mães adolescentes e entre aqueles que não moram com ambos os pais chama a atenção. Certamente, a concentração da gravidez na adolescência em grupos sociais menos favorecidos e o pouco preparo da mulher neste ciclo de vida para a maternidade contribuem para o acúmulo de experiências adversas neste subgrupo ${ }^{62,63}$. Já o maior número de adversidades entre adolescentes de famílias monoparentais ou que convivem com madrastas e padrastos parecem estar associados ao fato de que estas famílias são mais propensas a infligir maus tratos contra as crianças ${ }^{32,64}$.

A negligência emocional foi a experiência adversa que mais se correlacionou com as demais, principalmente com as três formas de abuso (emocional, físico e sexual) e com a negligência física. Segundo alguns autores ${ }^{11,65}$, além da coocorrência de abusos e negligências por parte dos responsáveis ser bastante comum, a negligência pode estar abrindo espaço para outras formas de abuso infantil não familiar, visto que a criança não está totalmente protegida ${ }^{66,67}$. A privação de cuidado, afeto e proteção, somada às diferentes formas de abuso durante à infância justamente por aqueles que deveriam prover um ambiente seguro, traz várias consequências negativas no curto, médio e longo prazo. Dentre estas, destacam-se as lesões físicas, baixa autoestima, dificuldades em estabelecer relacionamentos sociais, comportamento agressivo, isolamento, sofrimento de ordem psíquica, entre outros ${ }^{66,67}$. A situação é ainda mais dramática ao se perceber que, além da negligência e dos abusos, quase $10 \%$ da amostra também experimentou ausência de pelo menos um genitor, o que pode reduzir ainda mais a segurança afetiva da criança, além de diminuir a supervisão e o orçamento familiar, podendo prejudicar ainda mais o crescimento e desenvolvimento da criança ${ }^{55,56}$.

Outro ponto que merece destaque, diz respeito às características dos adolescentes que não sofreram qualquer tipo de EAI. Como apontado previamente, os subgrupos que parecem estar mais protegidos ou menos propensos às EAI neste estudo são os adolescentes filhos de mães não adolescentes no momento de seu nascimento e aqueles que moram com ambos os pais. Outros estudos também destacam que a proteção familiar à criança, manifestada através de ações que demonstremenvolvimento e monitoramento dos pais nas atividades dos filhos com amigos ea residência em bairros seguros, está associada a resultados mais favoráveis na saúde e diminuição da exposição das crianças às EAI ${ }^{68,69}$.

Ressalta-se ainda que a escola é um espaço privilegiado para o enfrentamento das EAI. Seu papel é especialmente importante na detecção precoce dos casos, na notificação de situações que mereçam ações dos serviços de proteção, no acolhimento das crianças, adolescentes e suas famílias visando a redução do problema e, sobretudo, no desenvolvimento de estratégias de fortalecimento da resiliência para suportar as adversidades já vivenciadas ${ }^{70}$.

Os resultados deste estudo devem ser vistos à luz de suas limitações. Dentre estas, destacase o fato de que algumas adversidades, como o ambiente familiar disfuncional, problemas de saúde mental, abuso de álcool/drogas, familiar encarcerado, entre outras, não foram investigadas. Outro ponto é que, por ser um estudo de base escolar, a pesquisa pode não ter incorporado situações mais graves com uma concomitância ainda maior de adversidades que tenham culminado na evasão escolar. Certamente, tanto a incorporação destas situações à lista de experiências adversas, como a inclusão de adolescentes que não frequentam mais a escola aumentaria ainda mais a prevalência das situações. 
Alguns pontos fortes também devem ser ressaltados. Trata-se de uma pesquisa com amostra representativa dos alunos do Ensino Médio de escolas públicas e privadas de uma RA do município do Rio de Janeiro com características socioeconômicas e demográficas semelhantes a maior parte do município, o que possibilita postular que os resultados do estudo possam ser generalizados para uma população mais ampla. A utilização do instrumento QUESI, reconhecido internacionalmente por suas boas propriedades psicométricas também é um ponto positivo do estudo. A opção pelo autopreenchimento dos questionários visando reduzir o constrangimento do respondente certamente contribuiu para a validade das estimativas de prevalência dos agravos. $\mathrm{O}$ fato da entrevista ter ocorrido durante a adolescência e não na idade adulta, como ocorre na maior parte das pesquisas sobre o tema, também é um aspecto positivo, já que minimiza a lacuna temporal entre a ocorrência e a apuração dos eventos, reduzindo a possibilidade de viés de memória ${ }^{13}$. O estudo também inova ao apresentar o perfil de coocorrência das EAI, pois a maioria das pesquisas anteriores se restringem à contabilização do número acumulado de EAI a que os indivíduos são expostos.

Quanto à heterogeneidade das estimativas de prevalência das EAI nos diversos estudos, a nosso ver, os principais motivos para tamanhas diferenças são as diferentes definições utilizadas e experiências investigadas; os diferentes instrumentos de aferição usados para identificação das EAI; os diferentes períodos de recordatório e a diversida- de de populações que participam das pesquisas em termos de seu perfil socioeconômico e de vulnerabilidade. Tais diferenças devem ser consideradas ao se comparar resultados de pesquisas já realizadas.

\section{Conclusão}

Este estudo apontou uma alta magnitude de EAI, sejam isoladas ou concomitantes, corroborando com estudos anteriores nacionais e internacionais que já indicavam que o abuso, a negligência e outras adversidades na infância são experiências frequentes e fortemente correlacionadas. $\mathrm{O}$ acúmulo de situações de vulnerabilidade potencializa suas consequências negativas na saúde, no comportamento, no desenvolvimento emocional, na sociabilidade, no desempenho escolar e profissional e em tantos outros aspectos necessários a uma plena existência cidadã. Desta forma, enfatiza-se a necessidade de se incorporar políticas de prevenção intersetoriais das EAI, especialmente aquelas voltadas à redução da incidência de violências de quaisquer naturezas contra crianças e ao acolhimento e apoio às famílias em situação de violência já instaladas. Neste contexto, a atuação em rede é fundamental para que as crianças que sofreram adversidades possam se desenvolver física e emocionalmente, dominar as tarefas educacionais e se desenvolver dentro da sociedade, rompendo o ciclo das experiências adversas nas próximas gerações.

\section{Colaboradores}

L Stochero foi responsável pela análise de dados e redação do artigo, CL de Moraes foi responsável pela concepção do estudo, redação e análise crítica do artigo, ES Marques, ME Reichenheim e ST Taquette foram responsáveis pela redação do artigo e revisão crítica final, EB dos Santos e DL Pacheco pela revisão da análise dos dados. 


\section{Financiamento}

O presente trabalho foi realizado com apoio da Coordenação de Aperfeiçoamento de Pessoal de Nível Superior (CAPES) (001), Fundação Carlos Chagas Filho de Apoio à Pesquisa do Estado do Rio de Janeiro (FAPERJ), Cientista do Nosso Estado (CNE) e Conselho Nacional de Desenvolvimento Científico e Tecnológico (CNPq).

\section{Referências}

1. Felitti VJ, Anda RF, Nordenberg D, Williamson DF, Spitz AM, Edwards V, Koss MP, Marks JS. Relationship of childhood abuse and household dysfunction to many of the leading causes of death in adults. The Adverse Childhood Experiences (ACE) Study. Am J Prev Med 1998; 14(4):245-258.

2. Kessler RC, McLaughlin KA, Green JG, Gruber MJ, Sampson NA, Zaslavsky AM, Aguilar-Gaxiola S, Alhamzawi AO, Alonso J, Angermeyer M, Benjet C, Bromet E, Chatterji S, Girolamo Gde, Demyttenaere K, Fayyad J, Florescu S, Gal G, Gureje O, Haro JM, Hu CY, Karam EG, Kawakami N, Lee S, Lepine JP, Ormel J, Posada-Villa J, Sagar R, Tsang A, Ustun TB, Vassilev S, Viana MC, Williams DR. Childhood adversities and adult psychopathology in the WHO World Mental Health Surveys. Br J Psych 2010; 197(5):378-385.

3. Heidinger LS, Willson AE. The childhood roots of adult psychological distress: Interdisciplinary perspectives toward a better understanding of exposure to cumulative childhood adversity. Child Abuse Neglect 2019; 97:104136.

4. Friedman EM, Montez JK, Sheehan CM, Guenewald TL, Seeman TE. Childhood Adversities and Adult Cardiometabolic Health: Does the Quantity, Timing, and Type of Adversity Matter? J Aging Health 2015; 27(8):1311-1338.

5. Bellis MA, Hughes K, Leckenby N, Jones L, Baban A, Kachaeva M, Povilaitis R, Pudule I, Qirjako G, Ulukol B, RalevaM,Terzic N. Adverse childhood experiences and associations with health-harming behaviours in young adults: surveys in eight eastern European countries. Bull World Health Organ 2014; 92(9):641-655.

6. Soares AL, Howe LD, Matijasevich A, Wehrmeister FC, Menezes AM, Goncalves H. Adverse childhood experiences: Prevalence and related factors in adolescents of a Brazilian birth cohort. Child Abuse Negl 2016; 51:21-30.

7. Oladeji BD, Makanjuola VA, Gureje O. Family-related adverse childhood experiences as risk factors for psychiatric disorders in Nigeria. Br J Psychiatry 2010; 196(3):186-191.

8. Loxton D, Townsend N, Dolja-Gore X, Forder P, Coles J. Adverse Childhood Experiences and Healthcare Costs in Adult Life. J Child Sexual Abuse 2019; 28(5):511-525.

9. Kalmakis KA, Chandler GE. Adverse childhood experiences: towards a clear conceptual meaning. $J A d v$ Nurs 2014; 70(7):1489-1501.

10. Lacey RE, Minnis H. Practitioner Review: Twenty years of research with adverse childhood experience scores - Advantages, disadvantages and applications to practice. J Child Psychol Psych 2020; 61(2):116-130.

11. World Health Organization (WHO). International Society for Prevention of Child Abuse and neglect. Preventing child maltreatment: a guide to taking action and generating evidence. Geneva: WHO; 2006.

12. Halfon N, Larson $\mathrm{K}$, Russ $\mathrm{S}$. Why social determinants? Healthc Q 2010; 14(1):8-20.

13. Coelho BM, Andrade LH, Borges G, Santana GL, Viana MC, Wang YP. Do Childhood Adversities Predict Suicidality? Findings from the General Population of the Metropolitan Area of Sao Paulo, Brazil. PLoS One 2016; 11(5):e0155639. 
14. Wolke D, Lereya ST. Bullying and parasomnias: a longitudinal cohort study. Pediatrics 2014; 134(4):e1040-e1048.

15. Burke NJ, Hellman JL, Scott BG, Weems CF, Carrion VG. The impact of adverse childhood experiences on an urban pediatric population. Child Abuse Negl 2011; 35(6):408-413

16. Wyman PA, Moynihan J, Eberly S, Cox C, Cross W, Jin $\mathrm{X}$, Caserta MT. Association of family stress with natural killer cell activity and the frequency of illnesses in children. Arc Pediatr Adolesc Med 2007; 161(3):228234.

17. Goncalves H, Soares AL, Santos AP, Ribeiro CG, Bierhals IO, Vieira LS, HellwigNL, Wehrmeister FC, Menezes AM. Adverse childhood experiences and consumption of alcohol, tobacco and illicit drugs among adolescents of a Brazilian birth cohort. Cad Saude Publica 2016; 32(10):e00085815.

18. Forster M, Gower AL, McMorrisBJ, BorowskyIW. Adverse childhood experiences and school-based victimization and perpetration. $J$ Interpers Violence 2017:886260517689885.

19. London S, Quinn K, Scheidell JD, Frueh BC, Khan MR. Adverse Experiences in Childhood and Sexually Transmitted Infection Risk From Adolescence Into Adulthood. Sex Transm Dis 2017; 44(9):524-532.

20. McMahon EM, Corcoran P, Keeley H, Clarke M, Coughlan H, Wasserman D, Hoven CW, Carli V, Sarchiapone M, Healy C, Cannon M. Risk and protective factors for psychotic experiences in adolescence: a population-based study. Psych Med 2020:1-9.

21. Ports KA, Merrick MT, Stone DM, Wilkins NJ, Reed J, Ebin J, Ford DC. Adverse Childhood Experiences and Suicide Risk: Toward Comprehensive Prevention. Am J Prev Med 2017; 53(3):400-403.

22. Youssef NA, Belew D, Hao G, Wang X, Treiber FA, Stefanek M, Yassa M, Boswell E, McCall WV, Su S. Racial/ ethnic differences in the association of childhood adversities with depression and the role of resilience. $J$ Affect Disord 2017; 208:577-581.

23. Liu RT. Childhood Adversities and Depression in Adulthood: Current Findings and Future Directions. Clin Psychol (New York) 2017; 24(2):140-153.

24. Huang HP, Yan Shan Z, Chen S, Li M, Luo C, Gao H, Hao L, Liu L. Adverse childhood experiences and risk of type 2 diabetes: A systematic review and meta-a nalysis. Metabolism 2015; 64(11):1408-1418.

25. Hughes K, Bellis MA, Hardcastle KA, Sethi D, Butchart A, Mikton C, Jones L, Dunne MP. The effect of multiple adverse childhood experiences on health: a systematic review and meta-analysis. Lancet Public Health 2017; 2(8):e356-e366.

26. Rossen E, Cowan K. The role of schools in supporting traumatized students. Principal's Res Review 2013; 8(6):1-7.

27. Sege RD, Harper Browne C. Responding to ACEs With HOPE: Health Outcomes From Positive Experiences. AcadPediatr 2017; 17(7S):S79-S85.

28. Cronholm PF, Forke CM, Wade R, Bair-Merritt MH, Davis M, Harkins-Schwarz M, PachterLM, Fein JA. Adverse childhood experiences: expanding the concept of adversity. Am J Prev Med 2015; 49(3):354-361.
29. Finkelhor D, Shattuck A, Turner H, Hamby S. A revised inventory of Adverse Childhood Experiences. Child Abuse Negl 2015; 48:13-21.

30. Prefeitura do Rio de Janeiro. Data.Rio - Informações sobre a cidade do Rio de Janeiro. Instituto Pereira Passos 2017 [acessado $2018 \mathrm{dez} 28$ ]. Disponível em: http://www.data.rio/

31. Arya R, Antonisamy B, Kumar S. Sample size estimation in prevalence studies. Indian J Pediatrics 2012; 79(11):1482-1488.

32. Krug EG, Mercy JA, Dahlberg LL, Zwi AB. The world report on violence and health. Lancet 2002; 360(9339):1083-1.088.

33. Bernstein DP, Fink L. Childhood Trauma Questionnaire: a Retrospective Self-Report manual San Antonio. TX: The Psychological Corporation; 1998.

34. Grassi-Oliveira R, Stein LM, Pezzi JC. Tradução e validação de conteúdo da versão em português do Childhood Trauma Questionnaire. Rev Saude Publica 2006; 40:249-255.

35. Associação Brasileira de Empresas de Pesquisa (Abep). Critério Padrão de Classificação Econômica Brasil. Abep; 2016. [acessado $2018 \mathrm{dez} 28$ ]. Disponível em: http://www.abep.org/criterio-brasil.

36. Kendall MG. A New Measure of Rank Correlation. Biometrika 1938; 30(1/2):81-93.

37. Stata Statistical Software. Release 13 [computer program]. Version 15. College Station, TX: StataCorp LP; 2015.

38. Organização das Nações Unidas (ONU). Convenção Internacional de Direitos da Criança. Genebra: ONU; 1989.

39. Brasil. Lei no 8.069 , de 13 de julho de 1990. Dispõe sobre o Estatuto da criança e do adolescente e dá outras providências. Diáriooficial da União 1990; 13 jul.

40. Knudsen EI. Sensitive periods in the development of the brain and behavior. J CognNeurosci 2004; 16(8):1412-1425.

41. Brodski SK, Hutz CS. Novas perspectivas sobre o abuso emocional. Diaphora 2016; 16(1):13-19.

42. World Health Organization (WHO). Preventing youth violence: an overview of the evidence. Genebra: WHO; 2015.

43. Moody G, Cannings-John R, Hood K, Kemp A, Robling M. Establishing the international prevalence of self-reported child maltreatment: a systematic review by maltreatment type and gender. BMC Public Health 2018; 18(1).

44. Brodski SK, Hutz CS. Novas perspectivas sobre o abuso emocional. Diaphora 2016; 16 (1):13-19.

45. Brown RC, Plener PL, Braehler E, Fegert JM, Huber -Lang M. Associations of adverse childhood experiences and bullying on physical pain in the general population of Germany. J Pain Res 2018; 11:3099-3108.

46. Arruda Silva P, Lunardi V, Lunardi G, Braga Arejano C, Stiff Ximenes A, Ribeiro J. Violência contra crianças e adolescentes: características dos casos notificados em um Centro de Referência do Sul do Brasil. Enfermería Global 2016; 16(46): 406-444. 
47. Malta DC, Antunes JT, Prado RR de, Assunção AA Freitas MI de. Fatores associados aos episódios de agressão familiar entre adolescentes, resultados da Pesquisa Nacional de Saúde do Escolar (PeNSE). Cien Saude Colet 2019; 24(4):1287-1298.

48. Minayo MCS. Violência contra crianças e adolescentes: questão social, questão de saúde. Rev Bras Saude Materno Infantil 2001; 1:91-102.

49. Dube SR, Anda RF, Whitfield CL, Brown DW, FelittiVJ, Dong M, Giles WH. Long-term consequences of childhood sexual abuse by gender of victim. Am J Prev Med 2005; 28(5):430-438.

50. Pinheiro PS. World report on violence against children. Geneva: WHO; 2006.

51. Cunningham TJ, Ford ES, Croft JB, Merrick MT, Rolle IV, Giles WH. Sex-specific relationships between adverse childhood experiences and chronic obstructive pulmonary disease in five states. Int J Chron Obstruct Pulmon Dis 2014; 9:1033-1042.

52. Soares Vieira M. Violência sexual contra meninas: do silêncio ao enfrentamento. Revista Libertas 2018; 18(2):101-116.

53. Connell-Carrick K. A Critical Review of the Empirical Literature: Identifying Correlates of Child Neglect. Child Adolesc Social Work J 2003; 20(5):389-425.

54. Pasian MS, Faleiros JM, Bazon MR, Lacharité C. Negligência infantil: a modalidade mais recorrente de maus-tratos. Pensando famílias 2013; 17:61-70.

55. Demarzo MMP. Dinâmica familiar, morte dos pais e saúde da criança. J Human Growth Develop 2011; 21:755-758.

56. Atrash HK. Parents' Death and its Implications for Child Survival. Rev Bras Crescimento Desenvolv Hum 2011; 21(3):759-770.

57. Feitosa EFMA. A incidência da alienação parental na família monoparental. Conteúdo Juridico [periódico na Internet] 2016. [acessado 2019 out 17] Disponivel em: https://conteudojuridico.com.br/consulta/Artigos/47543/a-incidencia-da-alienacao-parental-na-familia-monoparental

58. da Silva MR. Família Monoparental na atualidade e seus fatores determinantes. [monografia] Marília-SP: Fundação de Ensino Eurípides Soares da Rocha; 2016.

59. Giacomozzi A, Negrão N. A separação e disputa de guarda conflitiva e os prejuízos para os filhos. Liberabit 2015; 1:103-114.

60. Schabbel C. Relações familiares na separação conjugal: contribuições da mediação. Psicologia: teoria $e$ prática 2005; 7:13-20.

61. Valentim de Sousa DHA, Dias CMdSB. Recasamento: percepções e vivências dos filhos do primeiro casamento. Estudos de Psicologia 2014; 31:191-201.

62. Sidebotham P, Heron J. Child maltreatment in the "children of the nineties": a cohort study of risk factors. Child Abuse Negl 2006; 30(5):497-522.

63. Stith SM, Liu T, Davies LC, Boykin EL, Alder MC Harris JM, Jennifer M, Som A, McPherson M, Dees JEMEG. Risk factors in child maltreatment: A meta -analytic review of the literature. Aggres Violent Behavior 2009; 14(1):13-29.
64. Rodrigues LS, Chalhub AA. Contextos familiares violentos: da vivência de filho à experiência de pai. Pensando famílias 2014; 18:77-92.

65. Santana RP, Santana JSS. Violência contra criança e adolescente na percepção dos profissionais de saúde. Revista Enfermagem UERJ 2016; 24(4)e7070.

66. Gershoff ET. Corporal punishment by parents and associated child behaviors and experiences: a meta-analytic and theoretical review. Psychol Bull 2002; 128(4):539-579.

67. McLaughlin KA, Sheridan MA, Lambert HK. Childhood adversity and neural development: deprivation and threat as distinct dimensions of early experience. Neurosci Biobehav Rev 2014; 47:578-591.

68. Giacomozzi AI, Itokasu MC, Luzardo AR, Figueiredo CDSd, Vieira M. Levantamento sobre uso de álcool e outras drogas e vulnerabilidades relacionadas de estudantes de escolas públicas participantes do programa saúde do escolar/saúde e prevenção nas escolas no município de Florianópolis. Saude Soc 2012; 21:612622.

69. Moore KA, N. Ramirez A. Adverse childhood experience and adolescent well-being: do protective factors matter? Child Indicators Res 2016; 9(2):299-316.

70. Almeida SFC, Santos MCAB, Rossi TMF. Representações sociais de professores do ensino fundamental sobre violência intrafamiliar. Psicologia: Teoria e Pesquisa 2006; 22:277-286.

Artigo apresentado em 29/10/2019

Aprovado em 22/06/2020

Versão final apresentada em 24/06/2020

Editores-chefes: Romeu Gomes, Antônio Augusto Moura da Silva 
